\title{
Modelado y predicción a corto plazo del consumo y producción de energía eléctrica en una micro-red utilizando métodos basados en series temporales y redes neuronales artificiales
}

\author{
César Hernández, Francisco Rodríguez, José Carlos Moreno \\ Departamento de Informática, Campus de Excelencia Internacional Agroalimentario ceiA3, Centro de Investigación \\ en Energía Solar CIESOL, Universidad de Almería, 04120, España, e-mail: \{chdezh,frrodrig,jcmoreno\}@ual.es \\ Paulo Renato da Costa Mendes, Julio Elias Normey-Rico \\ Universidad de Santa Catarina, Florianopolis, Brasil, e-mail: paulorcm@hotmail.com, julio.normey@ufsc.br
}

\section{Resumen}

La previsión de la demanda eléctrica juega un papel clave en el funcionamiento de los sistemas de energía, ésto se debe a que la generación de energía por medio de fuentes renovables y sistemas distribuidos está creciendo en muchos países. Un análisis y modelado de la potencia eléctrica de los sistemas que conforman una Micro-red $(M G)$ se presenta en este trabajo. El sistema que se estudia consiste en una $M G$, esta $M G$ se encuentra integrada por un sistema fotovoltaico $(P V)$, un edificio bioclimático, un invernadero, un vehículo eléctrico y una interconexión con la red eléctrica que permite la compra y venta de energía. Este trabajo se centra en el estudio de cómo los métodos basados en series temporales y redes neuronales se pueden utilizar para las predicciones de la demanda de energía. El principal objetivo de los métodos presentados en este documento es predecir el comportamiento futuro a corto plazo. Se proporcionan comparaciones entre los diferentes métodos de predicción de energía.

Palabras clave: Modelado, Predicción, Demanda de energía, Series Temporales, Redes Neuronales.

\section{Introducción}

Existen muchos métodos para estimar la demanda de energía y que pueden ser caracterizados por la longitud del horizonte de predicción y la metodología seleccionada. El horizonte de predicción puede variar dependiendo de la aplicación y puede ser considerado como predicción a corto plazo, ésto es, menor de 60 minutos ([10], [6]) o predicción a largo plazo, ésto es, valores de predicción a lo largo de horas, días o meses. El uso de modelos que se basan en series temporales es una forma de estimar el comportamiento futuro a lo largo de un horizonte de predicción, estos modelos se basan en el supuesto de que los datos modelados se correlacionan y se caracterizan por tendencias y variaciones estacionales, tales como los muy conocidos modelos ARMA, ARIMA, ARMAX and ARIMAX ([3]). Por otro lado, las redes neuronales artificiales (ANN) proporcionan también una buena solución para realizar estimaciones debido a que su diseño se basa en el entrenamiento y no se necesitan supuestos estadísticos de los datos de origen. Las redes neuronales han sido ampliamente aceptadas como una herramienta para predecir series temporales, éstas ofrecen una forma alternativa para resolver problemas complejos ([9]). Las redes neuronales y los modelos ARIMA han sido comúnmente comparados en términos de la capacidad de predicción. Como una herramienta para sistemas no lineales, las redes neuronales NARX han sido exitosamente aplicadas a un gran numero de problemas de modelado de entrada-salida, tales como, sistemas biomédicos ([4]), la predicción de la comunicación de redes de tráfico ([2]), la demanda de energía en edificios ([11]).

En este artículo, el consumo y la producción de energía eléctrica de los sistemas que integran una MG es estudiado. Principalmente, este artículo se centra en el modelado y predicción de la producción de energía eléctrica de un sistema fotovoltaico y en el consumo de dos sistemas de carga.

Este artículo se organiza de la manera siguiente: en la sección 2, se presentan los sistemas que conforman la MG, además, se presenta la teoría de los modelos que se ha usado y los criterios de rendimiento; en la sección 3, se presentan los resultados y discusiones; finalmente, en la sección 4 se presentan las conclusiones.

\section{Materiales y métodos}

La MG que se estudia en este artículo está integrada por un sistema fotovoltaico (PV), un vehículo eléctrico del cual sus baterías se usan como un sistema de almacenamiento, y dos sistemas de carga (un edificio y un invernadero). En la Figura 1 se muestra esta micro-red.

\subsection{Materiales}

El invernadero estudiado en este artículo tiene una estructura tipo "Parral", una área de superficie de $877 \mathrm{~m}^{2}$, orientado en dirección N-S. Las instala- 


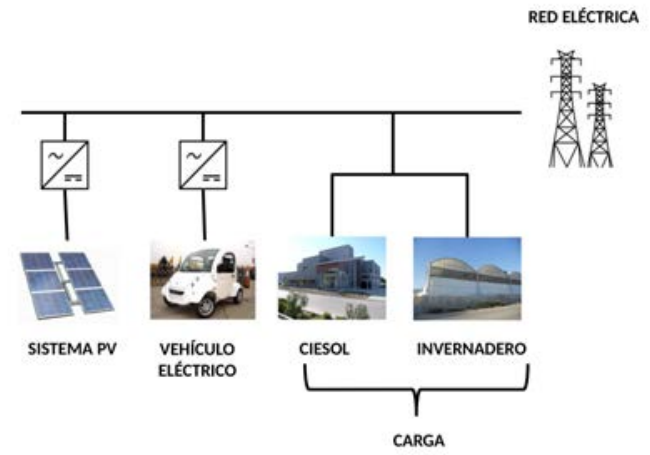

Figura 1: Micro-red.

ciones se encuentran ubicadas en la estación experimental Las Palmerillas de la Fundación Cajamar, en el municipio de El Ejido en el sureste de España [13]. El invernadero cuenta con sistema de ventilación lateral y cenital accionada por motores AC independientes, aerotermo, sistema de calefacción por tuberías de agua caliente alimentadas con biomasa, un sistema de enriquecimiento de $\mathrm{CO}_{2}$ creado por la quema de biomasa, mallas de sombreo y sistemas de alimentación de agua y nutrientes. El invernadero cuenta también, con un equipo de medida de 52 variables y está diseñado para el desarrollo de pruebas de identificación e implementación de estrategias de control climático. Además, cuenta con un sistema sensores de demanda de energía (SINEAX M 561) [14].

El edificio CDdI-CIESOL-ARFRISOL es un centro de investigación en energía solar localizado dentro del Campus de la Universidad de Almería en el sureste de España. Está distribuido en dos pisos con una superficie de $1071.92 \mathrm{~m}^{2}$, se compone por seis oficinas, todas las oficinas tienen orientación este y están situadas en la planta baja, con la excepción de la oficina principal, que se encuentra en el piso superior, ocho laboratorios que se orientan al norte (cuatro situados en la planta baja y dedicados a "Química Solar", y los otros cuatro se colocan en el piso superior, dos para el modelado y la unidad de control automático, y el restante para la evaluación de la unidad de recursos solares), una planta donde se encuentra una maquina de alta eficiencia de calefacción y absorción, y por último, cuenta también con una cocina y el cuarto de servicios.

El sistema PV se encuentra localizado en la terraza del edificio CIESOL. Es un sistema de $9.324 k W p$, con un número total de 4 módulos asociados en conjuntos de 14 paneles con una capacidad de $222 \mathrm{Wp} /$ panel, este sistema se encuen- tra conectado a 3 inversores de $2500 \mathrm{~W}$.

Las baterías con las que cuenta el vehículo eléctrico son del tipo de modelo Green Saver, con una capacidad total de $C=11 \mathrm{kWh}$.

\subsection{Métodos}

\subsubsection{Modelos NARMAX}

Los modelos NARMAX son una generalización no lineal de los modelos conocidos como ARX, los cuales constituyen una herramienta estándar en la identificación de modelos lineales de caja negra. Diferentes casos especiales de modelos NARMAX se presentan en [17]. En [5] se resumen estos modelos:

- $N A R\left(n_{y}\right)$ modelo No lineal Auto-Regresivo:

$$
y(t)=F\left[y(t-1) \ldots, y\left(t-d_{y}\right)\right]+e(t)
$$

- NARMA $\left(d_{y}, d_{e}\right)$ modelo No lineal AutoRegresivo con Media móvil:

$$
\begin{aligned}
& y(t)=F\left[y(t-1) \ldots, y\left(t-d_{y}\right), e(t-1),\right. \\
& \left.\ldots, e\left(t-d_{e}\right)\right]+e(t)
\end{aligned}
$$

- $N A R X\left(d_{y}, d_{u}\right)$ modelo No lineal AutoRegresivo con entradas exógenas:

$$
\begin{aligned}
& y(t)=F\left[y(t-1) \ldots, y\left(t-d_{y}\right), u(t-1)\right. \\
& \left.\ldots, u\left(t-d_{u}\right)\right]+e(t)
\end{aligned}
$$

donde $y, e$ y $u \in \mathbf{R}$ se refiere a las salida, el ruido y las entradas externas del sistema modelado, respectivamente, $d_{y} \geq 1, d_{x} \geq 1, d_{u} \leq d_{y}$ se refiere a el máximo retraso en las salidas, el ruido y las entradas, respectivamente, y $F$ es una función suave. De manera general, existe una gran equivalencia entre los modelos NARX y las redes neuronales recurrentes [16].

\subsubsection{Criterios de rendimiento de los modelos}

Con la finalidad de evaluar el desempeño de los modelos que se presentan en este trabajo, diferentes parámetros estadísticos han sido usados, estos parámetros de criterio de evaluación han sido mencionados en [8],[12]: el error cuadrático medio (MSE), la raíz del error cuadrático medio (RMSE) y el coeficiente de determinación $R^{2}$. Estos se definen como:

$$
M S E=\frac{1}{n} \sum_{k=1}^{n}\left(\hat{y}_{k}-y_{k}\right)^{2}
$$




$$
\begin{aligned}
R M S E & =\sqrt{\frac{1}{n} \sum_{k=1}^{n}\left(\hat{y}_{k}-y_{k}\right)^{2}} \\
R^{2} & =\frac{\sum_{k=1}^{N}\left(\hat{y}_{k}-\bar{y}\right)^{2}}{\sum_{k=1}^{N}\left(y_{k}-\bar{y}\right)^{2}}
\end{aligned}
$$

donde $\bar{y}$ es el valor de la media aritmetica de la señal medida.

\section{Resultados}

Este artículo se centra principalmente en la predicción a corto plazo de la demanda y producción de energía eléctrica. La predicción de la demanda tiene grandes ventajas para la administración de energía en micro-redes. En la Tabla 1 se muestran los principales valores estadísticos, el consumo y producción de energía durante los periodos que se mencionan más adelante de los sistemas que se estudian en este trabajo. Para el caso del edificio CIESOL sólo ha sido considerado la potencia demandada por un laboratorio. Los datos que se han recolectados de todos los subsistemas han sido previamente filtrados usando el filtro de Savitzky-Golay [15], este filtro preserva las características de la distribución inicial, así como el máximo y mínimo relativo y el ancho de los picos.

\subsection{El invernadero.}

Con la finalidad de modelar el consumo del invernadero, un modelo basado en redes Bayesianas ha sido presentado en [7], donde el consumo se ha clasificado en cuatro clases: bajo, medio, alto y muy alto. El principal problema de tener el consumo dividido en clases es que se pierde mucha información sobre el consumo real. Para evitar este tipo de problemas, en este artículo se propone un modelo ARX. El consumo eléctrico ha sido considerado como un sistema MISO (múltiples entradas una salida), donde la temperatura interior, $v_{1}(k)$, la temperatura exterior, $v_{2}(k)$, la humedad relativa interior, $v_{3}(k)$, la radiación global, $v_{4}(k)$, han sido consideradas perturbaciones del sistema. La soplante (inyección de $\mathrm{CO}_{2}$ ), $u_{1}(k)$, bomba de calefacción, $u_{2}(k)$, las aperturas de la ventilación cenital y lateral, $u_{3}(k)$ y $u(k)$, respectivamente, han sido consideradas como entradas del sistema y el consumo eléctrico, $y(k)$, ha sido considerado la salida del sistema.

Después de realizar varias pruebas, se ha observado que modelos del tipo ARX como sigue:

$$
\begin{aligned}
& A\left(z^{-1}\right) y(k)=\sum_{i=1}^{4} z^{-d_{B_{i}}} B_{i}\left(z^{-1}\right) u_{i}(k) \\
& +\sum_{i=1}^{4} z^{-d_{D_{i}}} D_{i}\left(z^{-1}\right) v_{i}(k)+\frac{\epsilon(k)}{\Delta}
\end{aligned}
$$

han presentado un buen ajuste a la dinámica del sistema real, para determinar estos modelos se ha usado el criterio de Información de Akaike. Se han calculado dos modelos, uno para representar la dinámica del consumo durante las estaciones de otoño-invierno y otro para representar la dinámica del consumo durante las estaciones de primavera-verano, esto se ha realizado con la finalidad de tener un mejor ajuste para las diferentes dinámicas del sistema, ya que el invernadero presenta consumos altos durante las estaciones otoñoinvierno y consumos bajos durante las estaciones primavera-verano. Para identificar los modelos, se han usado datos recolectados durante las estaciones primavera-verano y otoño-invierno del año 2014.

Tabla 1: Principales valores estadísticos de la potencia eléctrica $(k W)$ de cada subsistema durante el año 2014.

\begin{tabular}{l|c|c|c}
\hline $\begin{array}{l}\text { Valor } \\
\text { estadistico }\end{array}$ & Invernadero & $\begin{array}{c}\text { Edificio } \\
\text { CIESOL }\end{array}$ & $\begin{array}{c}\text { Sistema } \\
\text { PV }\end{array}$ \\
\hline Min & 0.073 & 4.486 & 0 \\
Mediana & 0.1071 & 6.265 & 0.397 \\
Media & 0.2678 & 6.342 & 2.282 \\
Max & 11.364 & 8.669 & 9.182 \\
Desviación & 0.7317 & 0.461 & 2.903 \\
estándar & & & \\
\hline
\end{tabular}

Se han obtenido los siguientes polinomios en tiempo discreto para el modelo ARX durante las estaciones otoño-invierno:

$$
\begin{aligned}
& A(z)=1-2.565 z^{-1}+2.225 z^{-2}-0.6589 z^{-3} \\
& D_{1}(z)=-0.0421 z^{-1}+0.08125 z^{-2}-0.05735 z^{-3} \\
& +0.01836 z^{-4} \\
& D_{2}(z)=0.02693 z^{-1}-0.05611 z^{-2}+0.03798 z^{-3} \\
& -0.009041 z^{-4} \\
& D_{3}(z)=-2.223 \times 10-5 z^{-1}+0.0001617 z^{-2} \\
& +0.0001483 z^{-3}-0.0002672 z^{-4} \\
& D_{4}(z)=1.802 \times 10^{-5} z^{-1}-4.474 \times 10^{-5} z^{-2} \\
& +5.606 \times 10^{-5} z^{-3}-2.685 \times 10^{-5} z^{-4} \\
& B_{1}(z)=-0.005899 z^{-1}+0.005135 z^{-2} \\
& +0.001135 z^{-3}+0.0006666 z^{-4} \\
& B_{2}(z)=0.007682 z^{-1}-0.004606 z^{-2} \\
& -0.0009907 z^{-3}+0.0003367 z^{-4} \\
& B_{3}(z)=2.699 \times 10^{-5} z^{-1}-7.67 \times 10^{-5} z^{-2} \\
& -2.764 \times 10^{-5} z^{-3}+5.427 \times 10^{-5} z^{-4} \\
& B_{4}(z)=4.117 \times 10^{-5} z^{-1}+4.485 \times 10^{-5} z^{-2} \\
& +1.851 \times 10^{-5} z^{-3}-8.345 \times 10^{-5} z^{-4}
\end{aligned}
$$




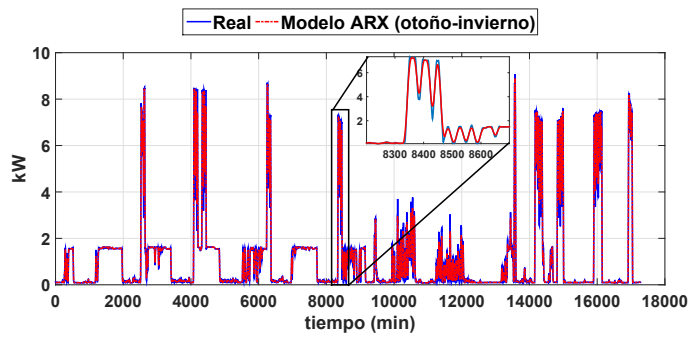

(a) Estaciones otoño-invierno

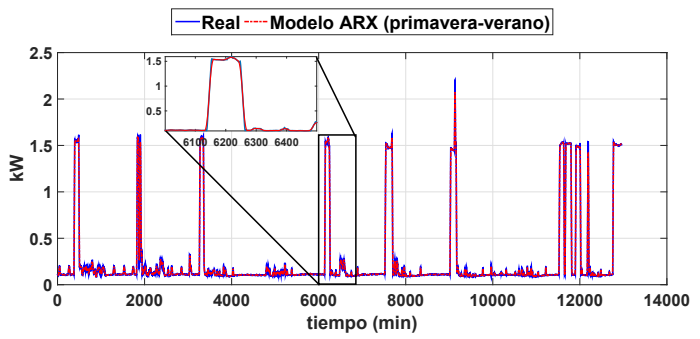

(b) Estaciones primavera-verano

Figura 2: Validación del modelo ARX para el invernadero.

así también, se han obtenido los siguientes polinomios en tiempo discreto para el modelo ARX durante las estaciones primavera-verano:

$$
\begin{aligned}
& A(z)=1-2.554 z^{-1}+2.592 z^{-2}-1.437 z^{-3} \\
& +0.4044 z^{-4} \\
& D_{1}(z)=-0.00916 z^{-1}+0.02126 z^{-2} \\
& -0.01735 z^{-3}+0.006083 z^{-4} \\
& -0.0008814 z^{-5} \\
& D_{2}(z)=0.002869 z^{-1}-0.004757 z^{-2} \\
& +0.003224 z^{-3}-0.001529 z^{-4} \\
& +0.0002556 z^{-5} \\
& D_{3}(z)=0.0002969 z^{-1}-0.0006606 z^{-2} \\
& +0.000631 z^{-3}-0.0003453 z^{-4} \\
& +8.325 \times 10^{-5} z^{-5} \\
& D_{4}(z)=1.287 \times 10^{-5} z^{-1}-1.866 \times 10^{-5} z^{-2} \\
& +1.055 \times 10^{-5} z^{-3}-6.092 \times 10^{-6} z^{-4} \\
& +6.234 \times 10^{-7} z^{-5} \\
& B_{1}(z)=0.0145 z^{-1}-0.01244 z^{-2} \\
& -0.005239 z^{-3}+0.002721 z^{-4} \\
& +0.004171 z^{-5} \\
& B_{2}(z)=0.001345 z^{-1}+0.004574 z^{-2} \\
& -0.00153 z^{-3}-0.003707 z^{-4} \\
& +0.007976 z^{-5} \\
& B_{3}(z)=-5.427 \times 10^{-6} z^{-1}-7.565 \times 10^{-7} z^{-2} \\
& +4.136 \times 10^{-6} z^{-3}+4.372 \times 10^{-6} z^{-4} \\
& -2.21 \times 10^{-6} z^{-5} \\
& B_{4}(z)=5.683 \times 10^{-6} z^{-1}-4.522 \times 10^{-6} z^{-2} \\
& -2.007 \times 10^{-6} z^{-3}-1.461 \times 10^{-6} z^{-4} \\
& +2.557 \times 10^{-6} z^{-5}
\end{aligned}
$$

Con la finalidad de verificar el desempeño de los modelos, éstos han sido validados durante el año
Tabla 2: Parámetros de rendimiento en la validación de los modelos calculados para el invernadero.

\begin{tabular}{l|c|c}
\hline Parámetros & $\begin{array}{c}\text { Modelos ARX } \\
\text { (otoño- } \\
\text { invierno) }\end{array}$ & $\begin{array}{c}\text { Modelos ARX } \\
\text { (primavera- } \\
\text { verano) }\end{array}$ \\
\hline MSE & 0.0178 & 0.0003 \\
RMSE & 0.1335 & 0.0186 \\
$R^{2}$ & 0.9942 & 0.9979 \\
\hline
\end{tabular}

2015. Como ejemplo se muestra en la Figura 2 la validación del modelo ARX durante algunos días en los que se representa la dinámica para las estaciones otoño-invierno y primavera-verano. Por otro lado, en la Tabla 2 se muestra los criterios de desempeño para cada modelo que se ha obtenido.

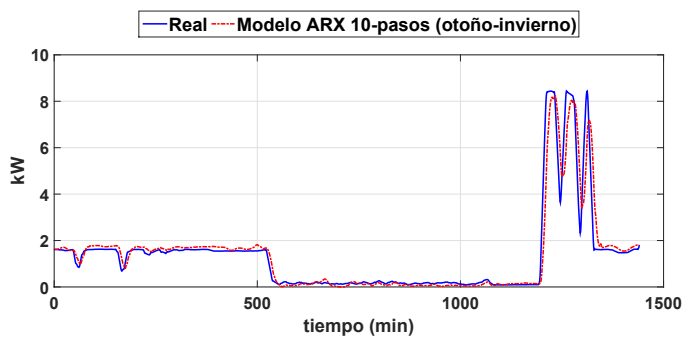

(a) Febrero 6 de 2015 con horizonte de predicción de 10 pasos

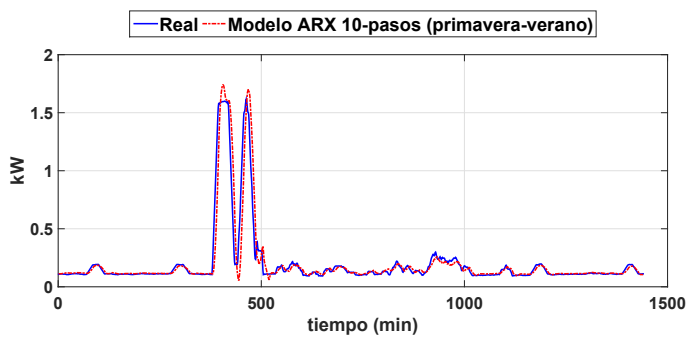

(b) Marzo 18 de 2015 con horizonte de predicción de 10 pasos

Figura 3: Resultados para los días 6 de febrero de 2015 y 18 de marzo de 2015 de los modelos ARX para el invernadero.

En la Figura 3 se muestra los resultados durante un día de otoño-invierno en la que se ha obtenido una predicción de 10 pasos. Los resultados del desempeño del modelo se muestran en la Tabla 3. Con la finalidad de calcular futuras predicciones, se ha considerado conocidas las futuras entradas, sin embargo, estas entradas pueden ser estimadas por otros modelos de predicción.

Como se muestra en la Figura 2 y en la Tabla 2, al realizar la validación de los modelos se puede observar que presentan una buena precisión de responder a los cambios, incluso al realizar una 
Tabla 3: Parametros de rendimiento de los modelos ARX para el invernadero en la predicción con un horizonte de 10 pasos.

\begin{tabular}{l|l|c}
\hline \multicolumn{3}{c}{ Horizonte de predicción de 10 pasos } \\
\hline Parámetros & $\begin{array}{l}\text { Modelo ARX } \\
\text { (otoño- } \\
\text { invierno) }\end{array}$ & $\begin{array}{c}\text { Modelo ARX } \\
\text { (primavera- } \\
\text { verano) }\end{array}$ \\
\hline MSE & 0.3419 & 0.0100 \\
RMSE & 0.5847 & 0.1000 \\
$R^{2}$ & 0.8969 & 0.8724 \\
\hline
\end{tabular}

predicción con un horizonte de 10 pasos como se muestra en la Figura 3 y en la Tabla 3. Los resultados de $R^{2}$ para este análisis han sido satisfactorios, ya que valores cercanos a 1 son deseados, se puede observar que se han alcanzado valores de 0.9942 y 0.9979 para los modelos de las estaciones otoño-invierno y primavera-verano, respectivamente. Adicionalmente, se puede observar que para las pruebas que se han realizado con horizonte de predicción de 10 pasos, también han sido satisfactorios.

\subsection{Edificio CIESOL y sistema PV.}

Con la finalidad de identificar modelos para la demanda del edificio CIESOL y la producción del sistema PV, se han elegido datos recolectados durante el año 2014 y se han identificado dos modelos para cada sistema.

Por un lado, una serie de pruebas en cada sistema para obtener un modelo tipo ARMA usando el criterio del error final de predicción (FPE) han sido realizadas, estos modelos se basan en un historial de datos de la potencia consumida y la potencia producida, respectivamente. Un modelo ARMA para una entrada - una salida basado en series temporales se representa por la siguiente ecuación:

$$
A\left(z^{-1}\right) y(k)=C\left(z^{-1}\right) e(k)
$$

La estructura de los modelos ARMA se reduce a la estructura de los modelos AR si $C\left(z^{-1}\right)=1$, como se puede ver en [1].

Por otro lado, para el mismo periodo, múltiples combinaciones de redes neuronales (NN) multicapa han sido evaluadas. Las combinaciones que se han realizado han incluido redes neuronales con diferentes números de capas ocultas, diferentes cantidad de unidades de neuronas en las capas y diferentes tipos de funciones de activación. Para obtener la red neuronal se ha realizado una serie de pruebas con las combinaciones que se han mencionado anteriormente, en cada combinación se ha minimizado el error $M S E$. Los resultados de la configuración de las redes neuronales para cada sistema han sido de una capa oculta con 11 y 10 neuronas para el edificio CIESOL y el sistema PV, respectivamente, además de una función sigmoide hiperbólica tangencial en la capa oculta, definida por:

$$
f(n)=\frac{1-e^{-n}}{1+e^{-n}}
$$

donde $n$ es peso de entrada en la capa oculta, y $f(n)$ es la función de activación en la salida de la capa oculta. Para la capa de salida, una función lineal de activación ha sido seleccionada, además de tener 8 y 6 neuronas en esta capa para el edificio CIESOL y el sistema PV, respectivamente.

Los polinomios en tiempo discreto que se han obtenido del modelo ARMA para la potencia consumida del edificio CIESOL son:

$$
\begin{aligned}
& A(z)=1-0.7436 z^{-1}-1.952 z^{-2}+1.304 z^{-3} \\
& +1.189 z^{-4}-0.7466 z^{-5}+0.059 z^{-6}-0.1033 z^{-7} \\
& -0.1601 z^{-8}+0.2462 z^{-9}+0.0057 z^{-10} \\
& -0.2231 z^{-11}+0.0506 z^{-12}+0.1216 z^{-13} \\
& -0.0127 z^{-14}-0.0353 z^{-15} \\
& C(z)=1+0.97 z^{-1}-0.8596 z^{-2}-0.9302 z^{-3} \\
& +0.014 z^{-4}-0.0020 z^{-5}+0.0060 z^{-6}+0.0137 z^{-7} \\
& -0.0023 z^{-8}+0.0064 z^{-9}+0.0145 z^{-10} \\
& -0.0022 z^{-11}+0.0057 z^{-12}+0.0138 z^{-13} \\
& -0.0023 z^{-14}-0.9932 z^{-15}-0.9551 z^{-16} \\
& +0.857 z^{-17}+0.9356 z^{-18}
\end{aligned}
$$

$\mathrm{y}$, los polinomios en tiempo discreto que se han obtenido del modelo ARMA para la producción del sistema PV son:

$$
\begin{aligned}
& A(z)=1-3.264 z^{-1}+4.55 z^{-2}-4.613 z^{-3} \\
& +4.43 z^{-4}-3.706 z^{-5}+3.049 z^{-6}-2.695 z^{-7} \\
& +2.638 z^{-8}-2.439 z^{-9}+1.595 z^{-10}-0.7346 z^{-11} \\
& -0.4916 z^{-12}+1.597 z^{-13}-1.226 z^{-14} \\
& +0.3109 z^{-15} \\
& C(z)=1-1.09 z^{-1}+0.3057 z^{-2}-1.278 z^{-3} \\
& +0.5316 z^{-4}+0.0166 z^{-5}+0.9545 z^{-6}-0.9729 z^{-7} \\
& +1.479 z^{-8}-1.004 z^{-9}+0.8663 z^{-10}-0.7941 z^{-11} \\
& -0.2833 z^{-12}-0.4224 z^{-13}+0.8239 z^{-14} \\
& -0.18951 z^{-15}+0.4131 z^{-16}-0.3577 z^{-17} \\
& -0.2445 z^{-18}+0.3576 z^{-19}-0.117 z^{-20}
\end{aligned}
$$

En la Tabla 4 se muestran los parámetros de validación durante el periodo desde el 4 hasta el 6 de junio de 2015. En este periodo han sido incluidos días laborales y no laborales, además, días soleados y días con nubes han sido incluidos (ver Figuras 4 y 5 ). 


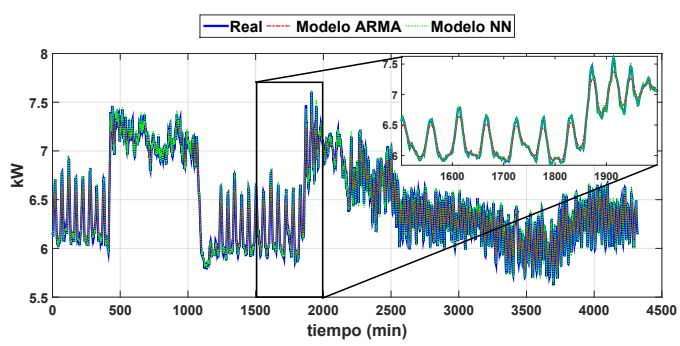

Figura 4: Validación de los modelo ARMA y NN para el edificio CIESOL en el periodo desde el 4 de junio hasta el 6 de junio de 2015 .

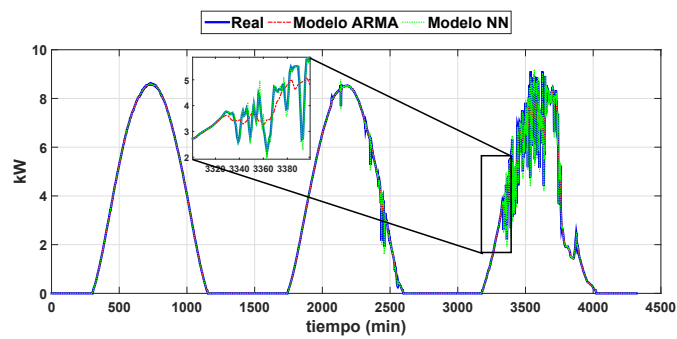

Figura 5: Validación de los modelo ARMA y NN para el sistema PV en el periodo desde el 4 de junio hasta el 6 de junio de 2015 .

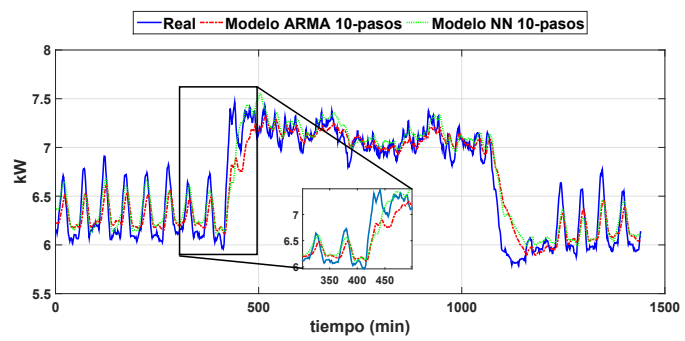

Figura 6: Resultados para el edificio CIESOL en el día 4 de junio de 2015 de los modelos ARX y $\mathrm{NN}$ con un horizonte de predicción de 10 pasos.

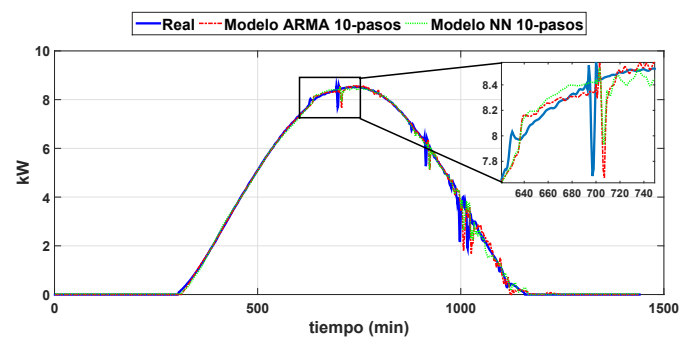

Figura 7: Resultados para el sistema PV en el día 5 de junio de 2015 de los modelos ARX y NN con un horizonte de predicción de 10 pasos.
Tabla 4: Parámetros de rendimiento en la validación de los modelos para el edificio CIESOL y el sistema PV.

\begin{tabular}{l|c|c}
\hline Parámetros & $\begin{array}{c}\text { Edificio } \\
\text { CIESOL }\end{array}$ & $\begin{array}{c}\text { Sistema } \\
\text { PV }\end{array}$ \\
\hline \multicolumn{3}{c}{ Modelo ARMA } \\
\hline MSE & 0.0054 & 0.0582 \\
RMSE & 0.0740 & 0.2412 \\
$R^{2}$ & 0.9690 & 0.9942 \\
\hline \multicolumn{3}{c}{ Modelo NN } \\
\hline MSE & 0.0003 & 0.0058 \\
RMSE & 0.0195 & 0.0765 \\
$R^{2}$ & 0.9978 & 0.9994 \\
\hline
\end{tabular}

Con la finalidad de evaluar la precisión de las predicciones, estos modelos han sido evaluados en predicciones con un horizonte de predicción de 10 pasos, estos resultados se muestran en las figuras 6,7 y en la Tabla 5 .

Tabla 5: Parámetros de rendimiento en la predicción con un horizonte de predicción de 10 pasos en los modelos para el edificio CIESOL y el sistema PV.

\begin{tabular}{l|c|c}
\hline Horizonte de predicción de 10 pasos \\
\hline Parámetros & $\begin{array}{c}\text { Edificio } \\
\text { CIESOL }\end{array}$ & $\begin{array}{c}\text { Sistema } \\
\text { PV }\end{array}$ \\
\hline \multicolumn{3}{c}{ Modelo ARMA } \\
\hline MSE & 0.0302 & 0.0404 \\
RMSE & 0.1739 & 0.2010 \\
$R^{2}$ & 0.8787 & 0.9962 \\
\hline \multicolumn{3}{c}{ Modelo NN } \\
\hline MSE & 0.0291 & 0.0346 \\
RMSE & 0.1708 & 0.1862 \\
$R^{2}$ & 0.8829 & 0.9967 \\
\hline
\end{tabular}

Para el edificio CIESOL y el sistema PV, de acuerdo a los resultados que se muestran en la Tabla 5, se puede observar que el modelo NN presenta mejores resultados (ver Figura 6). Se puede observar que el modelo NN aproxima mejor los picos de demanda. Para el sistema PV se puede observar en la Figura 7 que los dos modelos producen un pequeño desface en la predicción cuando hay nubes pasando.

\section{Conclusiones y trabajo futuro}

Este trabajo ha presentado el uso de diferentes métodos basados en series temporales. Estos métodos han sido usados para obtener una primera aproximación de las estimaciones futuras a corto plazo de la demanda y producción de energía eléctrica. Se han obtenido resultados satisfactorios. La principal ventaja del uso de estos 
métodos es el bajo coste computacional. Se ha podido estudiar el consumo del invernadero con más detalle, esto es, se ha corregido el problema que se presentaba al tener el consumo dividido en clases. En futuros trabajos se pretende realizar un modelo basado en redes neuronales para el invernadero y presentar una comparación con el modelo ARMAX obtenido en este trabajo. Por otro lado, se pretende estudiar con más detalle el consumo actual del edificio CIESOL y la producción de energía sistema PV. Estos modelos serán usados en combinación con técnicas de control predictivo con la finalidad de optimizar el uso de energía eléctrica en una micro-red.

\section{Agradecimientos}

Este trabajo ha sido financiado con el Proyecto $\mathrm{R}+\mathrm{D}+\mathrm{i}$ del Plan Nacional DPI2014-56364-C2-1$\mathrm{R}$ del Ministerio de Economía y Competitividad y Fondos FEDER.

\section{Referencias}

[1] Arahal, M. R., Soria, M. B., and Díaz, F. R. Técnicas de predicción con aplicaciones en ingeniería, vol. 15. Universidad de Sevilla, 2006.

[2] Bhattacharya, A., Parlos, A., And AtiYA, A. Prediction of MPEG-coded video source traffic using recurrent neural networks. IEEE Transactions on Signal Processing 51, 8 (Aug. 2003), 2177-2190.

[3] Contreras, J., Espinola, R., Nogales, F., AND Conejo, A. ARIMA models to predict next-day electricity prices. IEEE Transactions on Power Systems 18, 3 (Aug. 2003), 1014-1020.

[4] Coyle, D., Prasad, G., And McGinnity, T. M. A time-series prediction approach for feature extraction in a brain-computer interface. IEEE transactions on neural systems and rehabilitation engineering : a publication of the IEEE Engineering in Medicine and Biology Society 13, 4 (Dec. 2005), 461-7.

[5] GaO, Y., AND ER, M. J. NARMAX time series model prediction: feedforward and recurrent fuzzy neural network approaches. Fuzzy Sets and Systems 150, 2 (Mar. 2005), 331350.

[6] Gutiérrez-Alcaraz, G., Galván, E., GonzÁlez-CABrerA, N., AND JAVADI, M. Renewable energy resources short-term scheduling and dynamic network reconfiguration for sustainable energy consumption. $R e$ - newable and Sustainable Energy Reviews 52 (Dec. 2015), 256-264.

[7] Hernández, C., DEL Sagrado, J., Rodríguez, F., MOreno, J. C., AND SANCHEZ, J. A. Modeling of energy demand of a High-Tech greenhouse in warm climate based on Bayesian networks. Mathematical Problems in Engineering 2015 (2015), 11.

[8] Kaytez, F., Taplamacioglu, M. C., CAM, E., AND HARDAlac, F. Forecasting electricity consumption: A comparison of regression analysis, neural networks and least squares support vector machines. International Journal of Electrical Power \& Energy Systems 67 (May 2015), 431-438.

[9] Kouni, S., And Keynia, F. A new cascade NN based method to short-term load forecast in deregulated electricity market. Energy Conversion and Management 71 (July 2013), $76-83$.

[10] Massana, J., Pous, C., Burgas, L., MeLENDEZ, J., AND Colomer, J. Short-term load forecasting in a non-residential building contrasting models and attributes. Energy and Buildings 92 (Apr. 2015), 322-330.

[11] Mena, R., Rodríguez, F., Castilla, M., AND Arahal, M. A prediction model based on neural networks for the energy consumption of a bioclimatic building. Energy and Buildings 82 (2014), 142-155.

[12] Pawlowski, A., Guzman, J. L., RoDriguez, F., Berenguel, M., AND SANCHEZ, J. Application of time-series methods to disturbance estimation in predictive control problems. In 2010 IEEE International Symposium on Industrial Electronics (July 2010), IEEE, pp. 409-414.

[13] Rodríguez, F., Berenguel, M., Guzmán, J. L., AND RAmírez-Arias, A. Modeling and Control of Greenhouse Crop Growth. Advances in Industrial Control. Springer International Publishing, Cham, 2015.

[14] Sánchez-Molina, J. A., Reinoso, J. V., Acién, F. G., Rodríguez, F., And López, J. C. Development of a biomass-based system for nocturnal temperature and diurnal CO2 concentration control in greenhouses. Biomass and Bioenergy 67 (2014), 60-71.

[15] Savitzky, A., And Golay, M. J. Smoothing and differentiation of data by simplified least squares procedures. Analytical chemistry 36, 8 (1964), 1627-1639. 
[16] Sum, J., Kan, W.-K., And Young, G. A Note on the Equivalence of NARX and RNN. Neural Computing \& Applications 8, 1 (Mar. 2014), 33-39.

[17] Zemouri, R., Gouriveau, R., And ZerHOUNI, N. Defining and applying prediction performance metrics on a recurrent NARX time series model. Neurocomputing 73, 13-15 (Aug. 2010), 2506-2521. 